\title{
DEPRIVACIÓN SOCIOCULTURAL Y DESARROLLO DEL LENGUAJE EN NIÑOS DE LA V REGIÓN ${ }^{1}$
}

\author{
Ximena Sánchez Segura* \\ Francisco Fernández Mateo ${ }^{* *}$
}

\section{ANTECEDENTES GENERALES}

- is sociedades contemporáneas han mostrado una gran preocupación por desarrollar la educación, - - ocurando erradicar el analfabetismo y esforzándose en aumentar los niveles de escolaridad. En s países subdesarrollados estos esfuerzos estuvieron asociados a la creencia que la expansión de - educación sería un elemento importante, tanto para lograr el desarrollo como para combatir la breza. Las décadas de los años cincuenta y sesenta fueron en Iberoamérica buenos ejemplos de ta situación (García-Huidobro, Zúñiga.: 1990).

Aunque en dicho período se obtuvieron logros, a veces dignos de encomio, en cuanto al sremento en la cobertura y disminución del analfabetismo, los resultados en términos del ¿sarrollo y erradicación de la pobreza han sido, por decir lo menos, exiguos.

Toda sociedad moderna necesita de individuos con un nivel de escolaridad adecuado para implir con una serie de tareas necesarias para su funcionamiento. Un incremento generalizado $\therefore$ la escolaridad, debería aumentar las personas entrenadas disponibles para cumplir tales tareas. : $n$ embargo, se ha podido observar que un nivel similar de escolaridad no es equivalente a una milar calidad de educación: los sectores pobres de la población muestran los niveles más bajos $\therefore$ las mediciones de calidad educativa. Independiente de que la educación sea causa o efecto del S.sarrollo, si una sociedad le asigna alto valor a la escolaridad, quienes no alcancen determinados veles serán discriminados negativamente. Es posible plantear entonces, que la educación no sólo - Jede ser considerada como un insumo de producción, sino además y especialmente como un bien $\therefore$ consumo, como indicador de calidad de vida (Fliegel: 1981).

Es necesario señalar por lo tanto, que el desarrollo económico no aumenta automáticamente . cantidad o la calidad del desarrollo humano: "Cada sociedad debe adoptar por sí misma la : cisión de asignar recursos a la educación y a otras oportunidades de aprendizaje" (uNESCO, 1990: - . El desarrollo humano desde una perspectiva individual conlleva un proceso de aprendizaje y $\therefore$ aplicación de lo aprendido a mejorar la calidad de vida.

En la medida que las personas y los grupos tengan mayores conocimientos, podrán participar ¿ mejor manera en las tareas de construcción e interpretación de su sociedad, aportando nuevas 1eas para enfrentar los problemas y contribuyendo así a una mejor calidad de vida.

Sin embargo, la falta de oportunidades de aprendizaje limita no sólo el desarrollo de las - apacidades individuales sino, además, la capacidad de la sociedad para generar desarrollo y poder btener beneficio de los logros existentes.

Frente al problema del aprendizaje, es importante destacar que es en los grupos más pobres $\therefore$ la sociedad donde este proceso reviste particulares dificultades.

Las investigaciones en general señalan que toda actividad de aprendizaje depende fucrtemen¿ de las características de los educandos (unEsCo: 1990). Los indicadores más determinantes de

\footnotetext{
${ }^{1}$ Trabajo que forma parte del Proyecto FONDECYT 90-1282.

* Socióloga. Profesora Titular, Facultad de Educación, Universidad de Playa Ancha de Ciencias de la Educación.

**Sociólogo, Profesor Titular, Departamento de Sociología, Facultad de Ciencias Sociales, Universidad de Chile.
} 
las potencialidades de los niños que ingresan al ciclo de educación primaria son la salud, la nutrición y las condiciones del medio social en el que se desarrolla el niño. Diversos estudios permiten establecer diferencias en el coeficiente intelectual y en el rendimiento escolar en niños que pertenecen a las clases más bajas de la población comparativamente con niños de otras clases sociales (Filp: 1987; Bralic, Lira: 1983; Monckeberg: 1981). La mayoría de estos estudios han utilizado test psicométricos cuyos resultados establecen un rendimiento inferior en los niños de las clases bajas. El lenguaje se señala como una de las áreas más afectadas.

Es necesario señalar que el lenguaje es uno de los contenidos más importantes que el niño incorpora en la etapa de socialización primaria, no sólo posibilita todos los aprendizajes posteriores sino que marca y define las coordenadas de la vida cotidiana (Berger, Luckmann: 1973).

Gran parte de los trabajos orientados a la comparación de niños que pertenecen a distintos niveles socioeconómicos destacan tres aspectos que se interconectan en relación a la pobreza: La oportunidad de buscar metas y de solucionar problemas y la motivación existente para ello, lo que va a afectar también el uso que hace del lenguaje tanto para comunicarse como para procesar información. El segundo aspecto es especílicamente lingüístico: en la medida que el niño se expone a diferentes situaciones aprende a utilizar el lenguaje como control del pensamiento y como mecanismo de interacción social. El tercero tiene que ver con el modelo de reciprocidad hacia el cual se orienta el niño, que esperan de él sus padres, sus amigos y su comunidad. Al respec to es importante señalar que el aprendizaje del lenguajc a partir de un modelo es importante y representa un desafío para el niño, porque éste más que copiar conductas específicas del lenguaje ejercita reglas sobre cómo comportarse relacionadas con el lenguaje.

Sin embargo, no existen suficientes motivos para plantear que las influencias de un ambiente adverso en el proceso de desarrollo y de aprendizaje infantil sean del todo irreversibles. Es importante considerar, tanto la diversidad cultural como los aspectos universales referidos al desarrollo humano. Una teoría del desarrollo debe considerar ambas visiones, dado que es un modelo para comprender tanto la capacidad de perfección del ser humano, como su vulnerabilidad (Bruner, 1971).

\section{DESARROLLO Y APRENDIZAJE DEL LENGUAJE}

Se exponen a continuación en forma sumaria diferentes tcorías y enfoques sobre el aprendizaje, en los cuales es posible insertar el lenguaje como una conducta a desarrollar. Ellos permiten explicar comportamientos y aplicar estrategias a cualquier conducta susceptible de ser modificada.

\subsection{El Planteamiento de Bandura}

Interesa referirse aquí al aprendizaje por imitación de modelos, el cual en el marco de las teorías mediacionales enfatiza los aspectos sociales del aprendizaje en función de los principios conductistas. El aporte de Bandura es importante para las ciencias de la conducta y contribuye en el contexto sociológico a explicar aspectos del proceso de socialización primaria (Bandura, 1974).

Bandura en su teoría del aprendizaje social, intenta delinear un conjunto de principios referidos al aprendizaje con especial énfasis en las variables sociales, en la medida que se consideran importantes para explicar el desarrollo y la modificación de la conducta (Sánchez, 1988).

La imitación, según Bandura, es un aspecto fundamental del aprendizaje, pues aún cuando exista un estímulo para lograr una cercanía a la conducta esperada, la presencia de un modelo puede posibilitar la adquisición en menor tiempo. Se puede combinar la presencia del modelo con 
$\therefore$ refuerzo positivo lo cual traerá mejores resultados si además la conducta imitada es socialmente cptable. En el caso del aprendizaje del lenguaje, estudios plantean que "se adquiere y consolida $\therefore$ una matriz social en que su frecuencia se verá ostensiblemente aumentada en la medida que los - tores sociales que sirvan como agentes de cambio muestren una conducta lingüística constante:nte reforzada" (Sánchez, Villarroel, Dennis, 1989:15).

Si se considera que cada historia de vida es particular y única, no todos los niños son usceptibles en igual forma a los procesos de influencia social. Se plantea que niños dependientes -rán más influenciables y que niños con antecedentes de fracasos previos tenderán más a imitar los otros niños y a ser menos creativos, existiendo además diferencias por sexo, edad y - stenencia a la clase social (Bandura, 1974).

\section{- 2 El Enfoque de la Heurística}

Ėte planteamiento proviene del análisis de los procesos cognoscitivos y como éstos funcionan. ate del supuesto que la conducta lingüística opera con términos y categorías léxicas además de t tegorías sintácticas, pretendiendo enseñar el uso de reglas que faciliten el proceso de toma de zisiones en el sistema cognoscitivo frente a los estímulos verbales.

Aun cuando no es una teoría del lenguaje, parte del supuesto que el ser humano depende . sncialmente del uso del lenguaje para su desempeño social, por lo cual cualquier estrategia que - ztenda ayudar a desarrollarlo colaborará con un desarrollo integral del ser humano.

\section{-.3 El Planteamiento de Piaget}

Saget es considerado como un investigador en el campo de la epistemología genćtica. Su - cocupación se centra además en el desarrollo de los procesos intelectuales.

Plantea que en un comienzo las funciones cognoscitivas están ligadas al ejercicio de la cotricidad, pero aproximadamente a los dos años de edad surgen en el niño las primeras - spresentaciones simbólicas con las cuales trabajará el sistema cognoscitivo.

Primariamente la función simbólica aparece ligada a la motricidad del niño y se origina en las $1 v$ ariantes funcionales de acomodación y asimilación. El lenguaje es peculiar de cada sujeto y se ncuentra fuertemente ligado a la función sensorio motriz. En el proceso del desarrollo del niño ¿ ta función simbólica pierde su privacidad y empieza a hacerse colectiva, es en este punto donde 1 lenguaje se transforma fundamentalmente en un instrumento de comunicación colectiva.

Para Piaget el niño se enfrenta solo al mundo, especialmente al mundo físico en el que existen lgunas regularidades invariantes. Su tarea será construir una representación del mundo al cual se ¿ enfrentado lo que hará con la ayuda de un conjunto de propiedades lógicas que subyacen en su - insamiento y que corresponden a su estadio de desarrollo (Bruner, 1984).

A pesar de que Piaget no se refiere especialmente, ni se detiene en el problema del lenguaje vializado, aspecto desarrollado posteriormente por Bruner y Bernstein, plantea en diversos untos de su obra que el lenguaje como función simbólica, se relaciona c interactúa con la función .telectiva gencral potenciándola y perfeccionándola en su desarrollo. Se desprende de lo anterior importancia de la estimulación linguística que posibilitará un mayor desarrollo de las estructuras atclectivas en general (Sánchez, Villarroel, Demis 1989).

\section{-+ El Pensamiento de Bruner}

- teoría del desarrollo de Bruner constituye una altemativa importante frente a otras teorías : tuales, incorporando a la explicación psicológica de los procesos humanos su carácter social. 


\section{REVISTA DE SOCIOLOGÍA}

Desde la perspectiva de Bruner no es posible hablar de niños en "abstracto" sino de niños que crecen en ambientes específicos y determinados. Su obra tiene además fuertes referencias a las contribuciones de Vygotsky y Piaget por la repercusión e influencia de sus concepciones de la infancia.

Con respecto al desarrollo del lenguaje, Bruner plantea que es diferente la acción que establece el niño sobre los objetos que sobre las personas. Es en la acción sobre las personas donde se crean las primeras formas de comunicación preverbal. El niño no desarrolla las reglas de comunicación relativas a la adquisición del lenguaje en el vacío, las inserta en su relación con el mundo social.

Bruner plantea que las primeras interacciones tempranas que el niño realiza "tienen un carácter asimétrico, en las cuales el adulto tiene un papel determinante. El adulto tiene gran cantidad de medios de expresión mientras que el niño tiene muy pocos... Según Bruner, es esta práctica habitual de los adultos la que constituye la matriz interactiva en la que el pequeño podrá desarrollar su capacidad de comunicación significativa (Bruner, 1984:16).

La educación es, por lo tanto, fundamental para el desarrollo infantil, debido a que la capacidad operatoria del ser humano va a permitir la modularización de la acción, su descomposición y su reorganización en unidades superiores. Esta capacidad está derivada de la selección biológica, pero desarrollada por el entorno social.

La escuela con el desarrollo del lenguaje escrito es fundamental para el desarrollo cognoscitivo en la medida que posibilita la utilización del lenguaje fuera del contexto de una realidad inmediata.

\section{CLASE SOCIAL Y DESARROLLO DEL LENGUAJE}

Con relación a los antecedentes teóricos presentados y con especial referencia al planteamiento de Bruner, es posible señalar la importancia del mundo social en el desarrollo de las funciones intelectivas, especialmente aquellas que dicen relación con el lenguaje. Es por lo tanto factible plantear que existiría una dimensión de la desigualdad social, que diría relación con el desarrollo del lenguaje infantil en las clases más bajas de la sociedad. Es importante frente a lo señalado el planteamiento del sociólogo inglés Basil Bernstein.

\subsection{La Tesis de Bernstein}

El planteamiento central se refiere "tanto a la naturaleza, como a los procesos de transmisión cultural y al papel que juega en estos el lenguaje en los contextos de producción y reproducción, como el trabajo la familia y la educación" (Díaz, 1985:9). Su tesis está compuesta de dos planteamientos fundamentales a saber: Cómo las características de la clase social, regulan o determinan la estructura de la comunicación en la familia, y la orientación del código sociolingüístico de los niños, y cómo las características de la clase social regulan o determinan la institucionalización de los códigos elaborados en la educación (Díaz: 1985).

El análisis de Bernstein referido a los códigos de comunicación plantea entre otros aspectos, que el lenguaje del niño y el uso que éste hace de él en la clase baja no concuerda con las exigencias cognoscitivas y lingüísticas de la escuela, lo que constituye un obstáculo a la integración del niño al sistema formal de enseñanza y al éxito escolar (Bernstein, 1973).

Existirían según la tesis propuesta dos tipos de subsistemas lingüísticos o códigos que corresponderían respectivamente a las clases bajas y medias (Bernstein, 1973, 1990) a saber:

Código Restringido: presente en las clases bajas, contiene frases cortas, por lo general sin terminar, este lenguaje es pobre en símbolos, siendo básicamente descriptivo, concreto y tangible. 
Tiende a expresar desde el punto de vista sociolingüístico la solidaridad de grupo, tanto de los propios contenidos como de los medios formales que maneja. El aspecto cognoscitivo de este código no favorece el desarrollo de un nivel importante de implicacioncs lógicas, posee poco nivel de generalización y depende fuertemente de su contex to de referencia.

Código Elaborado: Presente en las clases medias, se caracteriza por el uso correcto de la sintaxis, la comunicación tiende a individualizar la expresión del pensamiento, en vez de reforzar la solidaridad colectiva. Desde el punto de vista sociolingüístico se presenta claramente como una forma o un medio de expresión individual: "El individuo se sitúa en un contex to de diálogo respecto a los otros miembros del grupo sin perder la conciencia de su individualidad... Desde el punto de vista cognoscitivo este tipo de código concede importancia a la organización racional del pensamiento, la planificación y la expresión explícita de la actividad mental" (Rondal, 1983:78).

Bernstein plantea además que las formas de socialización orientan al niño hacia códigos de habla diferente que controlan el acceso a significados relativamente dependientes o relativamente independientes del contexto: "los códigos elaborados orientan a sus usuarios hacia signilicados universalistas, mientras que los códigos restringidos orientan y sensibilizan a sus usuarios en significados particularistas: Estos dos códigos conducen a formas lingüísticas diferentes y reposan sobre relaciones sociales diferentes" (Bernstein, 1985:31). Es importante señalar además que según el autor, el niño de clase media a diferencia del niño de clase baja maneja ambos códigos: restringido y claborado, dependiendo del contexto situacional.

Bernstein, subraya que cuando él habla de código restringido, no quiere decir que los hablantes no utilicen en oportunidades variantes más elaboradas, lo que plantea es que estas formas de comunicación se utilizan con menor frecuencia, durante el proceso de socialización familiar (Bernstein, 1985).

"Podemos observar que la tesis de Bernstein constata un importante determinismo linguístico en los aprendizajes. Para Bernstein los modos de comunicación y las formas lingüísticas orientan el desarrollo intelectual y determinan todas las adquisiciones. El punto de vista según el cual el lenguaje determina todo o parte del desarrollo intelectual corresponde a escuelas psicológicas como la de Vygotsky y Bruner (Rondal, 1983: 86.87).

Con respecto a los antecedentes teóricos aquí señalados y a la problemática que evidencian, el equipo de investigación que representan los autores de este tabajo ${ }^{2}$, ha realizado y se encuentra realizando una serie de investigaciones cmpíricas en la V Región del país que se enmarcan en una línea de investigación referida al problema del desarrollo y uso del lenguaje en los niños pobres.

Se presentan a continuación algunos resultados de estos trabajos con el propósito de orientar la búsqueda de soluciones al deficitario desarrollo del lenguaje infantil en contextos deprivados.

\section{ESTIMULACIÓN Y DESARROLLO DEL LENGUAJE: DOS EXPERIENCIAS EN LA V REGIÓN}

La existencia de fuertes deterioros en el desarrollo del lenguaje infantil dificulta la participación del niño en el sistema formal de enseñanza, dado que el modelo de transmisión y evaluación del conocimiento en nuestro país es esencialmente verbal.

Considerando la importancia y la utilidad demostrada por la estimulación (Condemarín,

${ }^{2}$ Forma parte del equipo de investigación además de los autores de este trabajo, la profesora de la Universidad de Playa Ancha de Ciencias de la Educación, Sra. Gladys Villatroel Rosende. 
1986) para superar problemas en el lenguaje el equipo de investigación que representan los autores han asesorado dos experiencias en la V Región, basadas en la elaboración y puesta en marcha de programas de estimulación en contextos deprivados.

\subsection{Estimulación del lenguaje en niños deprivados sociocultural y afectivamente}

Se señalan aquí un conjunto de actividades de estimulación del lenguaje, en niños internos por medidas de protección simple del hogar de menores "René Pienovi" de la Comuna de Valparaíso.

En el caso del menor en situación irregular interno por medida de protección simple, existe una doble desventaja que afecta el desarrollo normal de las estructuras intelectivas y en especial el lenguaje: la situación de deprivación sociocultural originada por la pertenencia a las clases más bajas y la de deprivación afectiva, originada por el desarraigo familiar y la despersonalización que independiente de la intencionalidad de las instituciones responsables del cuidado de los niños, se vive en los hogares de menores.

Los datos que se presentan a continuación corresponden a 33 casos de niños de sexo masculino, cuyas edades fluctuaban entre 8 y 10 años, que asistían a $3^{\circ}$ y $4^{\circ}$ básicos en escuelas municipalizadas de Valparaíso.

\section{Cuadro 1 \\ CAMBIOS EN EL RENDIMIENTO VERBAL DE NIÑOS DESPUÉS DE UN PROGRAMA DE ESTIMULACIÓN}

\begin{tabular}{|l|cc|}
\hline $\begin{array}{l}\text { Tipo de rendimiento } \\
\text { psicolingǘstico }\end{array}$ & $\begin{array}{c}\text { Antes del } \\
\text { programa }\end{array}$ & $\begin{array}{c}\text { Después del } \\
\text { programa }\end{array}$ \\
\hline Armónico & 6 & 15 \\
Disarmónico & 27 & 18 \\
\hline Total & 33 & 33 \\
\hline
\end{tabular}

Los datos muestran que con posterioridad a las acciones de estimulación y reforzamiento del lenguaje, el rendimiento se incrementó sustancialmente. El rendimiento verbal fue medido mediante la aplicación de la batería de exploración verbal para trastornos del aprendizaje BEVTA (Bravo, Pinto 1987).

\subsection{Elaboración y Aplicación de Estrategias para estimular el Lenguaje en Niños Desnutridos}

Esta experiencia tuvo lugar en tres Centros de Atención de Desnutrición y Desarrollo del Lenguaje, denominados actualmente Jardines Faniliares de la comuna de Viña del Mar.

La labor de estos centros en el momento de la investigación era entregar una ración mejorada de alimentos y estimular el lenguaje de los niños con la colaboración de las madres. El trabajo realizado por el equipo de investigación estuvo centrado en la elaboración de tres estrategias metodológicas para estimular el lenguaje basadas en tres modelos teoricos de aprendizaje ${ }^{3}$.

${ }^{3}$ Al respecto: "Lenguaje y pobreza, estimulación del lenguaje en niños deprivados socioculturalmente". En Avanoss de Investigación, Universidad de Playa Ancha. Dirección General de Investigación. Universidad de Playa Ancha, 1988-1989. 
DEPRIVACIÓN SOCIOCULTURAL Y DESARROLLO DEL LENGUAJE EN NIÑOS DE LA V REGIÓN

Los modelos teóricos utilizados, estuvieron representados por los planteamientos de Bandura, Piaget y los principios generales de la heurística.

Las estrategias consistieron en un conjunto de planificaciones educativas de acuerdo al aodelo curricular utilizado por la Junta Nacional de Jardines Infantiles.

Las acciones de estimulación estuvieron a cargo de alumnas de la carrera de Educación ?arvularia de la Universidad de Playa Ancha de Ciencias de la Educación. El instrumento de `edición utilizado fue el Test de Desarrollo Psicomotor TEPsi (Haussler, Marchant, 1985).

De esta experiencia se presentan en el cuadro siguiente los principales resultados de las iciones de estimulación realizadas.

Cuadro 2

CAMBIO EN LOS NIVELES DE DESARROLLO DEL LENGUAJE EN NIÑOS DESNUTRIDOS DE ACULRDO A ACCIONES DE ESTIMULACIÓN BASADAS EN DIFERENTES MODLLOS DE APRENDIZAJE

Caso 1: Teoría de base Bandura

\begin{tabular}{|lcr|}
\hline Categorías del test & $\begin{array}{c}\text { Antes de la } \\
\text { estimulación }\end{array}$ & $\begin{array}{c}\text { Después de la } \\
\text { estimulación }\end{array}$ \\
\hline Nomal & 9 & 14 \\
Riesgo & 5 & 3 \\
Retraso & 4 & 1 \\
\hline Total & 18 & 18 \\
\hline
\end{tabular}

Caso 2: Teoria de base Piaget

\begin{tabular}{|lcc|}
\hline Categorías del test & $\begin{array}{c}\text { Antes de la } \\
\text { estimulación }\end{array}$ & $\begin{array}{c}\text { Después de la } \\
\text { estimulación }\end{array}$ \\
\hline Normal & 9 & 13 \\
Riesgo & 2 & 0 \\
Retraso & 2 & 0 \\
\hline Total & 13 & 13 \\
\hline
\end{tabular}

Caso 3: Teoría de base Heurística

\begin{tabular}{|lcc|}
\hline Categorías del test & $\begin{array}{c}\text { Antes de la } \\
\text { estimulación }\end{array}$ & $\begin{array}{c}\text { Después de la } \\
\text { estimulación }\end{array}$ \\
\hline Normal & 7 & 12 \\
Riesgo & 4 & 0 \\
Retraso & 1 & 0 \\
\hline Total & 12 & 12 \\
\hline
\end{tabular}


Como se observa en el cuadro 2 , los datos presentados permiten establecer la importancia de la estimulación del lenguaje en contextos deprivados.

Si bien es posible señalar que las acciones de estimulación al estar basadas en modelos de aprendizaje diferentes, pudiesen haber tenido énfasis distintos no por ello dejaron de lograr su objetivo central.

Es importante señalar además que en este caso se logró que un número importante de madres adscritas al programa cooperaran en las acciones de estimulación realizadas por las alumnas tesistas.

Las características de bajo costo de este programa, permiten aplicarlo a grupos mayores, privilegiando la teoría de aprendizaje con la cual los responsables de las acciones de estimulación estén más familiarizados.

\section{CLASE SOCIAL Y CÓDIGOS SOCIOLINGÜÍSTICOS: ALGUNOS ANTECEDENTES EMPÍRICOS EN LA V REGIÓN}

Durante los años 1990, 1991 y 1992 el equipo de investigación ha estado investigando la relación existente entre la pertenencia a la clase social y el uso de un código específico de comunicación.

Básicamente ha interesado validar algunos de los planteamientos centrales de las tesis de Bernstein referidos al uso específico de un código específico de comunicación por parte del niño según su pertenencia a una clase social determinada 4 . En este momento se dispone de un conjunto de datos producto de un estudio explicativo, cuyo objetivo central fue someter a validación empírica en un sector de la V Región los planteamientos teóricos de Bernstein ya señalados. Los procedimientos metodológicos utilizados para obtener la información de los datos que aquí se expondrán fueron los siguientes:

5.1 Se trabajó con una muestra al azar definitiva de 239 niños de ambos sexos, cuyas edades fluctuaban entre 8 años 6 meses y 10 años, los cuales no tenían problemas de aprendizaje y asistían a $4^{\circ}$ año básico en establecimientos municipalizados, particulares subvencionados y particulares pagados del radio urbano de las comunas de Valparaíso y Viña del Mar.

5.2 Para obtener los antecedentes que permitieron establecer el tipo de código usado por los niños, se seleccionaron dos láminas las cuales fueron presentadas en orden sucesivo al niño (primero una, después la otra), solicitándole en cada oportunidad que contase un cuento alusivo al tema de cada lámina.

5.3. La primera narración fue grabada y transcrita y posteriormente analizada mediante la técnica de análisis de contenido, utilizando cinco indicadores de orden sintáctico, los cuales presentan directa relación con la tesis de Bcrnstein referida al uso de los códigos sociolinguísticos.

5.4. Paralelamente fue aplicada a las madres de los niños o a sus madres sustitutas, una cédula de entrevista para obtener la información frente a la ocupación y nivel educacional del jefe de hogar, datos que se utilizaron para estratificar, además de información que complementó la investigación del proyecto FONDECY'T ya señalado, y que no forma parte de este trabajo a saber: modos de socialización familiar y exposición a medios de comunicación de masas. Siguiendo los planteamientos teóricos y metodológicos de Bernstein (1973-1990), se distinguieron cuatro clases: 1. Clase baja-baja, 2. Clase baja, 3. Clase media-baja y 4. Clase media y media-superior.

\footnotetext{
${ }^{4} \mathrm{Al}$ respecto: a) "Influencia de la estratificación social en la formación de los códigos sociolingüísticos y su relación con la percepción de los mensajes televisivos. Proyecto FONDECYT 90. 1282. b) "Código sociolingüístico y rendimiento escolar" Dirección General de Investigación. Universidad de Playa Ancha de Ciencias de la Educación, 1990. c) "Desarrollo del lenguaje en el contexto rural" Dirección General de Investigación. Universidad de Playa Ancha de Ciencias de la Educación, 1992.
} 
5.5 Validez y confiabilidad: Los instrumentos fueron aplicados durante el año 1990, a una jestra previa de 167 casos. Se trabajó además con validez de contenido para asegurarse que los : trumentos midieran el fenómeno estudiado, utilizándose también el juicio de expertos. De este iterial se presenta a continuación un cuadro que muestra la relación entre la clase social y el tipo código sociolingüístico utilizado por los sujetos en estudio.

Cuadro 3

TIPO DE CÓDIGO SOCIOLINGÜÍSTICO SEGÚN CLASE SOCIAL

\begin{tabular}{|c|c|c|c|c|}
\hline Tipo de código & $\begin{array}{c}\text { Baja-baja } \\
\%\end{array}$ & $\begin{array}{c}\text { Baja } \\
\% \\
\end{array}$ & $\begin{array}{c}\text { Media-baja } \\
\% \\
\end{array}$ & $\begin{array}{c}\text { Media } \\
\% \\
\end{array}$ \\
\hline surado & 27 & 41 & 43 & 59 \\
\hline _:ringido & 73 & 59 & 57 & 41 \\
\hline لid & 100 & 100 & $\begin{array}{l}100 \\
65\end{array}$ & $\begin{array}{l}100 \\
(51)\end{array}$ \\
\hline
\end{tabular}

cuadrado: 10.82 G.L. $3 \alpha: 0.012$.

Los datos muestran una marcada asociación entre la clase social y el uso prelerente de un Jigo específico de comunicación en la dirección señalada en el planteamiento de Bernstein: Las ses medias utilizan preferentemente código elaborado y las clases bajas, particularmente la se baja-baja, utilizan preferentemente código restringido.

Es importante señalar, además, la presencia de un número importante de casos de código - uringido en la muestra estudiada incluso en la clase media.

\section{CONCLUSIONES}

- s antecedentes expuestos permiten establecer las siguientes conclusiones generales:

6.1. Con respecto a la estimulación como un recurso para contribuir a superar deficiencias en . Jesarrollo del lenguaje en niños deprivados se señala lo siguiente:

a) Es posible elaborar y llevar a cabo actividades de estimulación del lenguaje razonablemente cotivas y de bajo costo.

b) Reconociendo las limitaciones con respecto al número de casos de las experiencias ...lizadas, lo que pone cautela en la apreciación de los resultados obtenidos, no puede negarse que : observa una tendencia clara y consistente con respecto al logro de los objetivos planteados: . var el nivel de desarrollo del lenguaje de los niños que participaron de los programas presenta- is.

c) Es importante señalar que en ambos casos se trataba de niños no sólo de contextos de breza, sino además con carencias afectivas en un caso y nutricional en otro. A pesar de estas - ficiencias que lesionan fuertemente las capacidades infantiles, se observaron importantes $\because$ gresos.

6.2. Lo señalado anteriormente no significa justificar la escasez de recursos existentes para snder y solucionar los problemas y necesidades educativas de los sectores más pobres de la ziedad. Muestra la posibilidad de llevar a cabo acciones y acumular experiencias, que puedan ntribuir a solucionar algunos de los graves problemas existentes con presupuestos limitados, intras se lucha por aumentar los recursos asignados a la educación y por una mejor distribución . : cllos que favorezca a los sectores más pobres, en especial a los niños. 
6.3. Referido al planteamiento teórico de Bernstein y a los datos presentados al respecto en este trabajo, es posible concluir lo siguiente:

a) La pauta construida para determinar algunos de los aspectos de la estructura del código en uso por los niños, permitió establecer dos tipos de código: Elaborado y Restringido. El primero se caracterizó como señala Bernstein y concordando con los indicadores seleccionados al respecto, como aquel código que presenta una mayor riqueza sintáctica la que se aprecia en la utilización variada de adjetivos y adverbios, el uso de oraciones complejas y concordantes, este tipo de código posee además un mayor tipo de implicaciones lógicas.

El segundo tipo de código se caracterizó por una menor riqueza sintáctica, poco uso de adjetivos y adverbios, y especialmente falta de oraciones complejas.

Como establece Bernstein este código sc identifica además por ser básicamente descriptivo y tangible.

b) De la muestra estudiada (239 relatos), se encontraron 138 casos que corresponden al uso de código restringido. El porcentaje más alto correspondió a la clase Baja-Baja: $73 \%$.

c) La asociación presentada entre las variables clase social y código sociolingüístico, permiten establecer que la clase se constituye en una variable explicativa del uso de un modo de comunicación específico.

Los datos expuestos, muestran una marcada asociación entre la clase social y el uso de un determinado tipo de código sociolingüístico en la dirección propuesta por Bernstein: La clase media utiliza preferentemente código elaborado y la clase baja-baja utiliza preferentemente código restringido.

d) Es necesario señalar además que los datos muestran una importante cantidad de casos de código restringido en las clases medias. Estas cifras ilustran un problema que no es privativo de las clases bajas, ameritando un estudio mís acabado del tema con el propósito de identificar las principales variables que estarían incidiendo en el fenómeno (Fernández, Sánchez, Villarroel: 1992).

6.4. Finalmente es necesario recordar que tal como ha sido planteado por UNEsCo, "En el mundo existe un consenso cada vez mayor que el desarrollo humano debe ser el centro de todo proceso de desarrollo, de que en tiempos de ajuste y austeridad económica es preciso proteger los servicios para los pobres, de que la educación, la capacitación de las personas mediante el suministro de niveles básicos de instrucción es verdaderamente un derecho humano y una responsabilidad social. Nunca antes se ha entendido mejor la naturaleza del aprendizaje y la educación básica en sus dimensiones psicológicas, culturales, sociales y económicas.

Los recursos financieros, tecnológicos y humanos de que se dispone a nivel mundial, para satisfacer las necesidades básicas de aprendizaje no tienen precedentes. Cuando estos factores se combinan con la reafirmación de la voluntad política de satisfacer las necesidades básicas del aprendizaje, el próximo decenio y el siglo venidero pueden verse como una oportunidad para alcanzar el desarrollo humano suficiente que permita enfrentar los reales graves desafíos que tendrá el mundo (UNESCO. 1990. Conferencia Mundial sobre Educación para Todos.)

\section{REFERENCIAS}

Bandura, A. (1974) Aprendizaje Social y Teoria de la Personalidad. Barelona. Alianza Editorial. Berger, P. LuCKMAnn, T. (1973) La Construcción Social de la Realidad. Buenos Aires. Editorial Amorrortu. Bernstein, B. $(1973,1990)$ Class, Code and Control. London.

(Primer semestre) (1985) Routledge, Kegan and Paul. En: Revista Colombiana de Educación.

Bralic, S. LirA, M. (1983) "Lactantes Chilenos Marginales, Aspectos Psicosociales de la Infancia Marginal en Chile". CIDR, Documento de Trabajo No 5. 


\section{DEPRIVACIÓN SOCIOCULTURAL Y DESARROLLO DEL LENGUAJE EN NIÑOS DE LA V REGIÓN}

Bravo, L. Pinto, J. (1987) Batería de Exploración Verbal para Trastornos de Aprendizaje. Santiago. CPEIP-Universidad Católica de Chile.

BrunNer, J. (1971) La Importancia de la Educación. Buenos Aires. Editorial Paidós.

(1984) Acción, Pensamiento y Lenguaje. Barcelona, Alianza Editorial.

CONDEMARÍN (1986) Madurez Escolar. Santiago. Editorial Andrés Bello.

M. DIA7. M. (1985) En: Revista Colombiana de Educación (Primer semestre).

Fernández, F., SÁnchez, X., Villarroel, G. (1992) Informe Final Proyecto fondecyt 90-1282. "Influencia de la

Estratificación en la Formación de los Códigos Sociolingüísticos y su Relación con la Percepción de los Mensajes

Televisivos". "Alfabetismo, Educación Primaria y Desarrollo Rural: Algunas preguntas y Algunas Respuestas

Tentativas". Santiago. En Amtmann C. Fernández F. (Eds.).

FLIEGEL, F. (1981) Comunicación y Desarrollo Rutral. Ediciones La Ciudad.

FiL., J. (1987) "El Párvulo Chileno y los Requerimientos para su Desarrollo" en CPu Desarrollo Infantil y Pobreza. Chile. Garcia-Huldobro, J. Zúñiga, L. (1990) ¿Qué Pueden Esperar los Pobres de la Educación? Santiago. Ediciones CIDE.

I Aaussler, M., Marchant, T. (1986) Test de Desarrollo Psicomotor. Santiago, Ediciones Universidad Católica de Chile.

Monckeberg G., Riumallo, J. (1981) El Programa de Centros Cerrados de Recuperación Nutricional en Chile: Una

Experiencia con la Marginalidad. Conin. Santiago.

RONDAL, J. (1983) Lenguaje y Educación. Barcelona, Editorial Médica y Técnica.

ذÁNCHEZ, X. (1988) "Aprendizaje Social. Principales Aspectos del Aprendizaje por Initación de Modelos". En: “Revista

Ciencias Sociales" $N^{\text {os }} 32-33$. Universidad de Valparáso.

SANChEZ, X., VILlarkoel, G., Dennis, E. (1989) "Lenguaje y Pobreza". En Avances de investigación. D.I.G.I. Universidad

de Playa Ancha de Ciencias de la Educación. Valparaíso.

SANCHEZ, X. et al. (1991) "Estimulación del Lenguaje en Niños Deprivados Afectiva y Socioculturalmente". En Estudios

Pedagógicos $\mathrm{N}^{\mathrm{Q}} 17$. Universidad Austral de Chile. Valdivia.

xesco (1990) Conferencia Mundial sobre Educación para todos. Jontien, Tailandia. 